\title{
Leakage radiation interference microscopy
}

\author{
Emiliano Descrovi, ${ }^{1}$ Elsie Barakat, ${ }^{2}$ Angelo Angelini, ${ }^{1,3}$ Peter Munzert, ${ }^{4}$ Natascia De Leo, ${ }^{3}$ \\ Luca Boarino, ${ }^{3}$ Fabrizio Giorgis, ${ }^{1}$ and Hans Peter Herzig ${ }^{2, *}$ \\ ${ }^{1}$ Dipartimento di Scienza Applicata e Tecnologia, Politecnico di Torino, C.so Duca degli Abruzzi 24, 10129 Torino, Italy \\ ${ }^{2}$ Optics \& Photonics Technology Laboratory, Ecole Polytechnique Fédérale de Lausanne (EPFL), rue A. L. Breguet 2 , \\ Neuchâtel CH-2000, Switzerland \\ ${ }^{3}$ Nanofacility, Istituto Nazionale di Ricerca Metrologica, Strada delle Cacce, 91, 10135 Torino, Italy \\ ${ }^{4}$ Fraunhofer Institute for Applied Optics and Precision Engineering IOF, Albert-Einstein-Str. 7, 07745 Jena, Germany \\ ${ }^{*}$ Corresponding author: hanspeter.herzig@epfl.ch
}

Received July 18, 2013; revised August 2, 2013; accepted August 3, 2013; posted August 7, 2013 (Doc. ID 194079); published August 26, 2013

\begin{abstract}
We present a proof of principle for a new imaging technique combining leakage radiation microscopy with high-resolution interference microscopy. By using oil immersion optics it is demonstrated that amplitude and phase can be retrieved from optical fields, which are evanescent in air. This technique is illustratively applied for mapping a surface mode propagating onto a planar dielectric multilayer on a thin glass substrate. The surface mode propagation constant estimated after Fourier transformation of the measured complex field is well matched with an independent measurement based on back focal plane imaging. (c) 2013 Optical Society of America

OCIS codes: (180.3170) Interference microscopy; (110.3175) Interferometric imaging; (240.6690) Surface waves. http://dx.doi.org/10.1364/OL.38.003374
\end{abstract}

Leakage radiation microscopy (LRM) is a well-known microscopy technique that is gaining more and more popularity because of its wide potential application in the fields of plasmonics and nano-optics [1]. LRM allows the direct imaging of a substrate leakage radiation indicative of the spatial distribution of evanescent modes coupled on a variety of photonic/plasmonic nanostructures [2-4]. In the past, a number of phenomena have been investigated by LRM, such as plasmon waveguiding [5], plasmon focusing [6], interference [7], and light chirality [], wherein only the field intensity is collected. Indeed, the spatial distribution of the phase of evanescent fields cannot be directly accessed by means of standard LRM.

In this Letter, we propose an imaging approach based on the combined use of LRM and a high-resolution interference microscope (HRIM) based on a Mach-Zehnder configuration [9]. The proposed arrangement expands the capabilities of more conventional LRM setups by providing both amplitude and phase information of (leaky) evanescent fields on transparent substrates.

The experimental setup is sketched in Fig. 1. A detailed description of the working principle of the interferometric technique employed for the amplitude and phase retrieval in this arrangement can be found in [10].

Here, a collimated beam from a frequency doubled Nd:YAG laser source at $\lambda=532 \mathrm{~nm}$ is split in two arms of a Mach-Zehnder interferometer by means of a polarizing beam splitter (PBS). The polarization of the light beams is controlled through a pair of half wavelength plates and Glann-Taylor polarizers. The so-called object beam is sent to an objective (numerical aperture, $\mathrm{NA}=0.5$ ) used for sample illumination. The effective NA of the illumination objective can be reduced as desired by means of a diaphragm placed in front of the objective entrance pupil.

The light emerging from the sample is collected by means of an oil immersion objective (NA = 1.4) and imaged through a tube lens on a charge-coupled device (CCD), where it is superposed with the reference beam.
The reference beam undergoes a set of five controlled phase shifts produced by means of a piezo-actuated mirror. An offline image-processing algorithm based on the Schwider-Hariharan method $[11,12]$ takes into account the five interference images recorded by the CCD and retrieves amplitude and phase distributions related to the collected field.

The experimental mounting is such that the illumination objective can be moved longitudinally (along the $z$ axis) with respect to the sample plane. Furthermore, the assembly of the illumination objective together with the sample holder stage can be laterally moved (along the $x$ and $y$ axes) with respect to the collection objective. This situation is depicted in more detail in Fig. 2(a). In the measurements presented below, the illumination and the collection objectives are positioned off-axis by a few tens of micrometers in order to reduce the collection of the direct illumination.

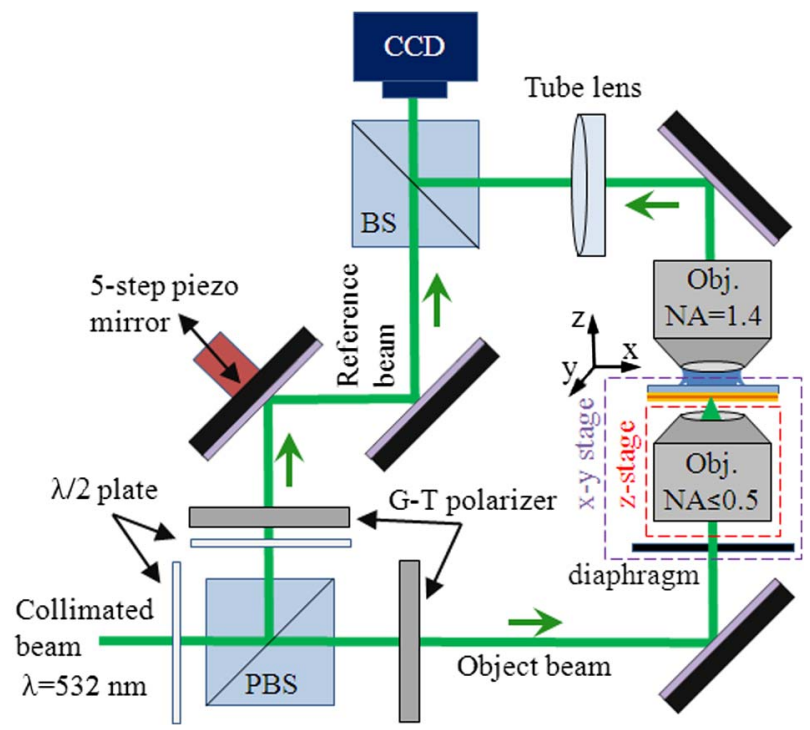

Fig. 1. Sketch of the optical paths in the HRIM used in leakage radiation configuration. 
The investigated structure consists of a $\mathrm{Ta}_{2} \mathrm{O}_{5}-\mathrm{SiO}_{2}$ multilayer [or one-dimensional photonic crystal (1-DPC)] deposited by ion beam assisted evaporation on a thin glass substrate [13]. As shown in Fig. 2(a), the dielectric stack is periodic, but the top silica layer is tailored, allowing a TE-polarized Bloch surface wave (BSW) to be coupled at a wavelength of $\lambda=532 \mathrm{~nm}$. Since BSWs are surface modes whose dispersion curves are located beyond the light line in air $[\underline{14}, \underline{15}]$, they can be coupled through a high index prism according to the Kretschmann configuration or, alternatively, by means of a grating coupler with proper period. In our case, since the illumination is provided from the air side of the 1-DPC, the use of a grating coupler is preferred. Such a linear grating is directly fabricated on the top silica layer by using focused ion beam (FIB) lithography over an area of $10 \mu \mathrm{m} \times 10 \mu \mathrm{m}$ approximately, as shown in Fig. 2(b). Atomic force microscopy measurements reveal a periodic corrugation with spatial period $\Lambda=460 \mathrm{~nm}$ and an average full depth $h=80 \mathrm{~nm}$.

When a Bertrand lens is inserted in the collection optical path before the tube lens, a back focal plane (BFP) image proportional to the Fourier transformation of the direct plane image [16] is produced on the CCD image plane [17]. This arrangement is particularly useful for collecting light belonging to the BSW leakage radiation as separated from the direct illumination and eventually the scattered light contributions. In Fig. 3(a) the BFP image of the collection oil immersion objective is presented. The external (green) circle defines the maximum NA of the objective $(\mathrm{NA}=1.4)$, while the inner (blue) circle defines the light line in air (corresponding to $\mathrm{NA}=1$ ). The direct incident illumination is visible as a circle with radius $\mathrm{NA}=0.15$, corresponding to the particular illumination configuration used in this case. When the object beam polarization is set as linearly polarized along the $x$ axis and the grating is brought under the illumination spot with its grating vector parallel to the $y$ axis, a bright arc appears beyond the air light line, at a location $k_{y} / k_{0}=1.19 \pm 0.2$. This is highlighted in Fig. 3(b), where the BFP intensity cross section along the red dotted line in Fig. 3(a) is plotted. The bright arc is polarized along the $x$ direction, and it is associated to a BSW coupled by the grating. As a check, the calculated TE-polarized angular reflectance profile at $\lambda=532 \mathrm{~nm}$ presented in Fig. 3(c) reveals the presence of BSW resonance dips at $k_{y} / k_{0}=1.15$ on the ideal multilayer layout from design [1].

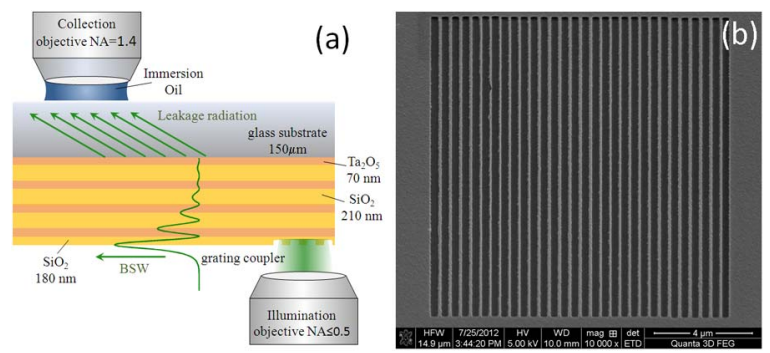

Fig. 2. (a) Detailed sketch of the photonic structure and the experimental arrangement used for sample illumination and leakage radiation collection. (b) Scanning electron microscope image of the grating coupler for a Bloch surface wave (BSW).

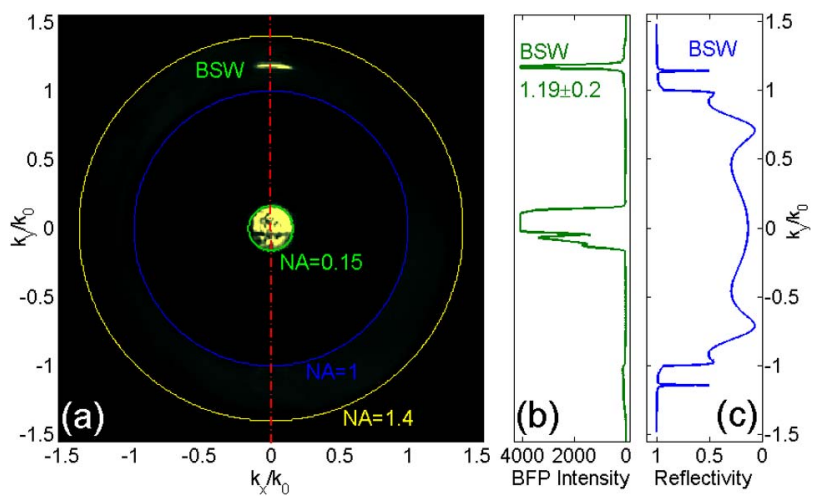

Fig. 3. (a) BFP image of grating coupled BSW upon illumination with a NA $=0.15$ beam. (b) Experimental cross-sectional intensity profile of BFP along the dashed red line in (a). (c) Calculated angularly resolved TE-polarized reflectance at $\lambda=532 \mathrm{~nm}$ revealing BSW resonances as indicated.

A recent paper [18] showed how LRM intensity images are affected by fringe modulations due to the coherent superposition of the leakage and the scattered radiation emitted from each of the plurality of point-like sources involved in an extended field distribution. As previously reported in Fig. 3, these two contributions can be rather well distinguished on the BFP, but they cannot be really separated on the direct plane image, and so interference effects occur.

In order to limit this effect, we managed to minimize the scattered light by positioning the grating (directly illuminated by the incidence beam) almost completely outside of the field of view of the collection objective and Fourier blocking almost completely the allowed light in the glass substrate. Since the 1-DPC surface has a very low roughness, we could limit such interference effects due to scattering. In these conditions, we performed a full amplitude/phase measurement through the five-step interferometric technique as described above. An exemplary result is presented in Fig. 4.

Specifically, Fig. 4(a) shows an amplitude distribution of the TE-polarized electric field associated to a BSW launched through direct illumination $(\mathrm{NA} \approx 0.25)$ of the grating, positioned at the top border of the image (saturated CCD pixels). The BSW propagates according to the arrow depicted in [19]. The presence of high-frequency fringes can barely be appreciated on the amplitude distribution, because of the above-mentioned effect considered in [18]. Moreover, Fig. 4(b) exhibits a corresponding BSW phase distribution, wherein the BSW propagation is revealed by a periodic sawtooth phase profile, as reported elsewhere [20]. By combining the amplitude and phase mappings, a complex electromagnetic field distribution can be obtained, whose real part is illustrated in Fig. 4(c).

If a fast Fourier transformation (FFT) is performed on the complex field recovered along the propagation direction, the spatial Fourier spectrum illustrated in Fig. 4(d) is obtained. The spatial spectrum is characterized by two peaks symmetrically centered at spatial frequencies $f_{y}^{\mathrm{BSW}}= \pm(2.25 \pm 0.3) \mu \mathrm{m}^{-1}$. The peak with positive frequency and higher amplitude is associated to a BSW propagating in the positive $y$ direction, while the peak with smaller amplitude is associated to a 

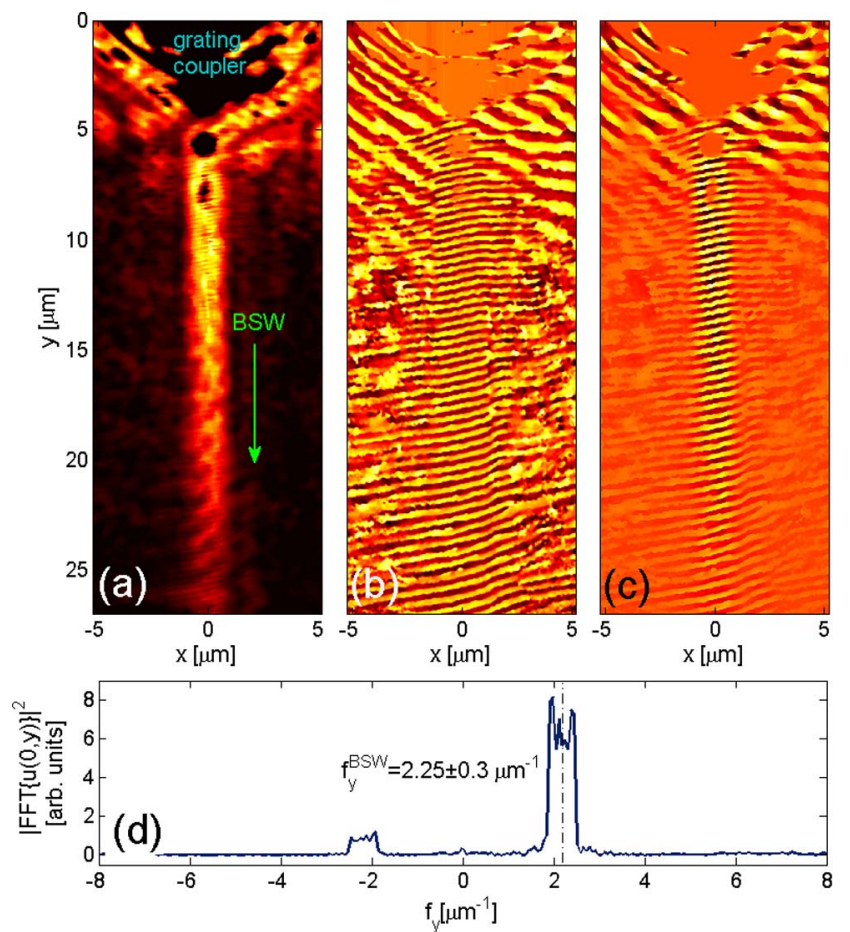

Fig. 4. (a) Direct plane image of BSW launched from a grating coupler. (b) Corresponding BSW phase distribution retrieved. (c) Real part of the obtained BSW complex field. (d) $|\mathrm{FFT}|^{2}$ profile of the measured complex field along the propagation direction of BSW.

BSW propagating in the negative $y$ direction, collected essentially from the grating region. The two detected peaks correspond to a spatial modulation of the complex field matching the BSW effective wavelength, as given by $\lambda_{\mathrm{eff}}^{\mathrm{BSW}}=\left|1 / f_{y}^{\mathrm{BSW}}\right|=444.4 \pm 59.3 \mathrm{~nm}$. This value should be compared with the effective BSW wavelength from the BFP image analysis in Fig. 3 as $\lambda_{\text {eff }}^{\mathrm{BSW}}=442.2 \pm 7.5 \mathrm{~nm}$, revealing a good matching between the two measurements. The larger uncertainty in the BSW effective wavelength as estimated from the direct-plane complex field FFT can be explained by a smaller spatial domain wherein the field can be properly detected.

In conclusion, we presented an original wide-field microscopy technique able to recover amplitude and phase distributions of evanescent fields that are leaky through a transparent substrate. This arrangement results from the combination of two well-known imaging techniques, namely LRM and HRIM. Upon the addition of an interferometric means according, e.g., to a MachZehnder configuration, a standard LRM can improve phase measurement capabilities in a rather easy way.

As a proof of principle, we demonstrated the effectiveness of this technique by imaging the complex field of a surface mode propagating on a proper photonic crystal. The TE-polarized BSW considered here allowed us to neglect some issues associated, e.g., to LRM imaging of TM-polarized evanescent fields [18]. In fact, in that case, contrast artifacts arising from the vectorial sum of TM-polarized light can likely be produced depending on the magnification of the imaging system employed. Moreover, taking into account the lateral resolution limited by diffraction, this interferometric leakage microscopy approach can be of great advantage in a number of nanophotonic characterizations where the phase information concerned with the evanescent fields is of major relevance.

The authors greatly acknowledge Dr. Emanuele Enrico (NanoFacility Piemonte, supported by Compagnia di San Paolo Foundation) for technical assistance. This research has received funding from the European Union Seventh Framework Programme (FP7/2007-2013) under grant agreement no. 318035—Project BILOBA, the Italian Flagship Project NANOMAX (Progetto Bandiera MIUR PNR 2011-2013), and the Swiss National Science Foundation (SNSF).

\section{References}

1. A. Drezet, A. Hohenau, D. Koller, A. Stepanov, H. Ditlbacher, B. Steinberger, F. R. Aussenegg, A. Leitner, and J. R. Krenn, Mater. Sci. Eng. B 149, 220 (2008).

2. C. J. Regan, A. Krishnan, R. Lopez-Boada, L. Grave de Peralta, and A. A. Bernussi, Appl. Phys. Lett. 98, 151113 (2011).

3. C. J. Regan, L. Grave de Peralta, and A. A. Bernussi, J. Appl. Phys. 111, 073105 (2012).

4. R. Wagner and F. Chicos, Phys. Rev. B 87, 165438 (2013).

5. S. Massenot, J. Grandidier, A. Bouhelier, G. Colas des Francs, L. Markey, J.-C. Weeber, A. Dereux, J. Renger, M. U. Gonzalez, and R. Quidant, Appl. Phys. Lett. 91, 243102 (2007).

6. T. Zentgraf, Y. Liu, M. Mikkelsen, J. Valentine, and X. Zhang, Nat. Nanotechnol. 6, 151 (2011).

7. V. Hafele, F. de Leon-Perez, A. Hohenau, L. Martin-Moreno, H. Plank, J. R. Krenn, and A. Leitner, Appl. Phys. Lett. 101, 201102 (2012).

8. Y. Gorodetsky, K. Y. Bliokh, B. Stein, C. Genet, N. Shitrit, V. Kleiner, E. Hasman, and T. W. Ebbesen, Phys. Rev. Lett. 109, 013901 (2012).

9. M.-S. Kim, T. Scharf, and H. P. Herzig, Opt. Express 18, 14319 (2010).

10. M. S. Kim, T. Scharf, C. Etrich, C. Rockstuhl, and H. P. Herzig, Opt. Lett. 37, 305 (2012).

11. J. Schwider, R. Burow, K.-E. Elssner, J. Grzanna, R. Spolaczyk, and K. Merkel, Appl. Opt. 22, 3421 (1983).

12. P. Hariharan, B. F. Oreb, and T. Eiju, Appl. Opt. 26, 2504 (1987).

13. A. Angelini, E. Enrico, N. De Leo, P. Munzert, L. Boarino, F. Michelotti, F. Giorgis, and E. Descrovi, New J. Phys. 15, 073002 (2013).

14. P. Yeh, A. Yariv, and C.-S. Hong, J. Opt. Soc. Am. 67, 423 (1977).

15. R. D. Meade, K. D. Brommer, A. M. Rappe, and J. D. Joannopoulos, Phys. Rev. B 44, 10961 (1991).

16. A. Berrier, M. Swillo, N. Le Thomas, R. Houdré, and S. Anand, Phys. Rev. B 79, 165116 (2009).

17. M. S. Kim, T. Scharf, C. Menzel, C. Rockstuhl, and H. P. Herzig, Opt. Express 20, 4903 (2012).

18. A. Hohenau, J. R. Krenn, A. Drezet, O. Mollet, S. Huant, C. Genet, B. Stein, and T. W. Ebbesen, Opt. Express 19, 25749 (2011).

19. E. Descrovi, T. Sfez, L. Dominici, W. Nakagawa, F. Michelotti, F. Giorgis, and H. P. Herzig, Opt. Express 16, 5453 (2008).

20. E. Descrovi, T. Sfez, M. Quaglio, D. Brunazzo, L. Dominici, F. Michelotti, H. P. Herzig, O. J. F. Martin, and F. Giorgis, Nano Lett. 10, 2087 (2010). 